\title{
2009 H1N1 Influenza Infection-Related Hospital Admissions: A Single Center Experience with Adult Patients in West Texas
}

\author{
Panupong Larppanichpoonphol, ${ }^{1}$ Hoda Mojazi Amiri, ${ }^{2}$ Taher Katouzian, ${ }^{3}$ \\ Rishi Raj, ${ }^{2}$ and Kenneth Nugent ${ }^{2}$ \\ ${ }^{1}$ Department of Internal Medicine, University of Rochester Medical Center, Rochester, NY 14642, USA \\ ${ }^{2}$ Department of Internal Medicine, Texas Tech University Health Sciences Center, Lubbock, TX 79430-9410, USA \\ ${ }^{3}$ Department of Internal Medicine, Louisiana State University Health Sciences Center, Shreveport, LA 71130-3932, USA
}

Correspondence should be addressed to Kenneth Nugent; kenneth.nugent@ttuhsc.edu

Received 25 April 2012; Accepted 21 June 2012

Academic Editors: F. Ferrero, D.-D. Ji, B. Kazemi, and N. Uchide

Copyright (C) 2013 Panupong Larppanichpoonphol et al. This is an open access article distributed under the Creative Commons Attribution License, which permits unrestricted use, distribution, and reproduction in any medium, provided the original work is properly cited.

\begin{abstract}
Background. Clinical information collected during the first wave of influenza pandemics may provide important projections about disease activity during subsequent waves. Methods. We performed a retrospective study of hospitalized patients with 2009 H1N1 influenza infection during the second wave and compared our findings with literature reports from the first wave. Results. There were 31 admissions, including 15 to the intensive care unit (ICU). Twenty-five patients (81\%) had at least one chronic medical condition; 12 patients (39\%) were obese. Thirty-three percent of the ICU patients and $75 \%$ of the non-ICU patients were admitted within 48 hours of symptom onset $(P=0.03)$. Infiltrates on CXR were seen in $60 \%$ of the ICU group and $19 \%$ of the non-ICU group within 48 hours of admission $(P=0.03)$. Forty-three percent of the ICU patients and $71 \%$ of the non-ICU patients received oseltamivir within 48 hours of illness. All non-ICU patients survived; $73 \%$ of the ICU patients survived $(P=0.043)$. Conclusions. Our patients in the second wave resembled patients reported from the first wave of the 2009 pandemic and had similar mortality rates. Delayed medical attention possibly explains the high number of ICU admissions in our study.
\end{abstract}

\section{Introduction}

The H1N1 influenza pandemic began in April 2009, and lasted until April 2010, in the USA. There were 61 million cases of $2009 \mathrm{H} 1 \mathrm{~N} 1$ infection in the USA, resulting in 274,000 hospitalizations and 12,470 deaths [1]. In contrast to seasonal influenza, more than $50 \%$ of cases, hospitalizations and deaths occurred in patients between 18 and 64 years of age [1-3]. During this pandemic, there were two waves of influenza activity in the USA [1]. The first wave started in mid-April and peaked in June; the second wave peaked in mid-October and declined quickly in December. Multiple waves of influenza activity have been observed in the past three pandemics, and the number of cases with pandemic influenza infection is usually higher after the first wave $[4,5]$. In addition, the second or third wave of the past pandemics is usually more lethal than the first wave [4-6]. Patients younger than 45 had a notably higher mortality rate than the elderly in all three waves of the 1918 pandemic [5-10]. Our hospital had no cases during the first wave but had multiple cases during the second wave. We report clinical information on hospitalized adults with $2009 \mathrm{H} 1 \mathrm{~N} 1$ influenza infection during the second wave and compare important outcomes to published information on the first wave. We also briefly discuss the community health implications of multiple waves of influenza activity.

\section{Materials and Methods}

Patient Population. We performed a retrospective study of patients diagnosed with $2009 \mathrm{H} 1 \mathrm{~N} 1$ influenza infection and admitted to University Medical Center (UMC), a 411-bed tertiary care hospital in Lubbock, Texas, from September 2009 to January 2010. This hospital is a referral center for West Texas and Eastern New Mexico and provides comprehensive 
TABLE 1: Epidemiologic characteristics and chronic medical conditions of hospitalized patients with $2009 \mathrm{H} 1 \mathrm{~N} 1$ influenza infection.

\begin{tabular}{|c|c|c|c|}
\hline Characteristic & $\begin{array}{c}\text { Total } \\
(n=31)\end{array}$ & $\begin{array}{c}\text { ICU } \\
(n=15)\end{array}$ & $\begin{array}{c}\text { Non-ICU } \\
(n=16)\end{array}$ \\
\hline Mean age (standard deviation) & $45(14)$ & $43(13)$ & $46(15)$ \\
\hline \multicolumn{4}{|l|}{ Age } \\
\hline$<20$ & 0 & 0 & 0 \\
\hline $21-40$ & $12(39 \%)$ & $7(47 \%)$ & $5(31 \%)$ \\
\hline $41-60$ & $16(51 \%)$ & $7(47 \%)$ & $9(56 \%)$ \\
\hline$>61$ & $3(9 \%)$ & $1(6 \%)$ & $2(13 \%)$ \\
\hline Female & $16(52 \%)$ & $9(60 \%)$ & $7(44 \%)$ \\
\hline BMI, mean (Standard deviation) & $31(11)$ & $33(15)$ & $30(6)$ \\
\hline $\mathrm{BMI}>30$ & $12(39 \%)$ & $6(40 \%)$ & $6(38 \%)$ \\
\hline Smoking & $9(29 \%)$ & $4(27 \%)$ & $5(31 \%)$ \\
\hline Alcohol use & $3(10 \%)$ & $1(7 \%)$ & $2(13 \%)$ \\
\hline No chronic medical condition & $6(19 \%)$ & $3(20 \%)$ & $3(19 \%)$ \\
\hline Hypertension & $21(68 \%)$ & $10(67 \%)$ & $11(69 \%)$ \\
\hline Diabetes & $13(42 \%)$ & $6(40 \%)$ & $7(44 \%)$ \\
\hline Chronic kidney diseases & $13(42 \%)$ & $4(27 \%)$ & $9(56 \%)$ \\
\hline Heart failure & $11(35 \%)$ & $5(33 \%)$ & $6(38 \%)$ \\
\hline Organ transplant & $7(23 \%)$ & $3(20 \%)$ & $4(25 \%)$ \\
\hline Pulmonary hypertension & $6(19 \%)$ & $1(7 \%)$ & $5(31 \%)$ \\
\hline Cancer & $4(13 \%)$ & $1(7 \%)$ & $3(19 \%)$ \\
\hline Coronary artery disease & $4(13 \%)$ & $2(13 \%)$ & $2(13 \%)$ \\
\hline COPD & $3(10 \%)$ & $2(13 \%)$ & $1(6 \%)$ \\
\hline Asthma & $2(6 \%)$ & $1(7 \%)$ & $1(6 \%)$ \\
\hline
\end{tabular}

${ }^{*}$ All $P$ values $>0.05$, BMI: Body mass index, and COPD: chronic obstructive pulmonary disease.

medical, surgical, pediatric, and obstetrical care. The 2009 H1N1 influenza infection was confirmed by either a CDCdeveloped reverse transcription polymerase chain reaction (RT-PCR) assay (Texas Department of State health Services, Austin, TX, USA) or a commercial RT-PCR assay (Quest Diagnostics, Madison, NJ, USA). We did not identify cases with positive RT-PCR results for $2009 \mathrm{H} 1 \mathrm{~N} 1$ influenza in the other months of 2009 and did not collect information on outpatients or children. We used records from the UMC clinical laboratory to identify patients with positive PCR results. The IRB for human studies of Texas Tech University Health Sciences Center approved the study with waiver of informed consent.

Data Collection. Data were collected for the following categories: epidemiologic characteristics, chronic medical conditions, clinical presentations, diagnostic laboratory, antiviral treatment, intensive care unit (ICU) admissions, and outcomes. Epidemiologic characteristics included age, sex, body mass index (BMI), smoking habit, and alcohol consumption. Chronic medical conditions included diabetes, hypertension, chronic kidney diseases, coronary artery disease, congestive heart failure, chronic obstructive pulmonary disease (COPD), asthma, pulmonary hypertension, organ transplant, and malignancy. Clinical presentations included date of the first symptom, date of admission, systemic symptoms, respiratory symptoms, gastrointestinal symptoms, vital signs, and oxygenation.

Diagnostic laboratory included complete blood counts and differentials, complete metabolic panels, RT-PCR assays for $2009 \mathrm{H} 1 \mathrm{~N} 1$ influenza on nasopharyngeal (NP) or oropharyngeal (OP) sample, chest X-rays, and blood cultures. Antiviral treatment included date of treatment initiation and duration of treatment. Intensive care unit admissions included time of admission, ventilator use, vasopressor use, and duration of ICU admission. Outcomes included diagnosis of acute respiratory distress syndrome (ARDS), septic shock, secondary bacterial infections, survival, and status at discharge.

Statistical Analysis. Statistical analysis was performed using SAS software (version 9.2, SAS Institute, Cary, NC, USA) and SPSS software (IBM SPSS version 19, Chicago, IL, USA). We report continuous variables as means and standard deviations and categorical variables as percentages. The $\chi^{2}$ test or Fisher's exact test was used for comparison of categorical variables. The Student's $t$-test was used for comparison of continuous variables. Logistic regression analysis was used to identify factors associated with ICU admission and with death. All statistical tests were two-sided, and comparisons at $P<0.05$ were considered significant.

\section{Results}

3.1. Clinical Information on All Admissions with 2009 H1N1 Influenza Infection. There were 31 hospital admissions for RT-PCR-confirmed 2009 H1N1 influenza infection between September 2009 and January 2010. Fifteen patients (48\%) were admitted to the ICU, and four (13\%) died. Fifty-two percent were female; the mean age was 45 years (Table 1 ). Twenty-five patients $(81 \%)$ had at least one chronic medical condition, including 12 patients (39\%) with a BMI greater than 30 (Table 1). Symptoms and selected vital signs are detailed in Table 2. Seventeen patients (55\%) presented to the hospital within 48 hours of the onset. Sixty-five percent had abnormal chest X-ray (Table 3). Ninety percent of the abnormalities were consistent with interstitial pneumonitis. Fiftyfive percent had lobar pneumonia based on their chest X-ray findings. The abnormalities on chest X-ray were identified within the first 48 hours of admission in 12 patients (39\%). The RT-PCR assay for $2009 \mathrm{H} 1 \mathrm{~N} 1$ influenza was positive in 28 NP samples and in 8 OP samples. Treatment, complications, and outcomes are detailed in Table 4 .

\subsection{Clinical Comparisons between ICU and Non-ICU Admis-} sions. There were no important differences in baseline characteristics between the ICU and non-ICU patients (Table 1). The mean time from the onset of illness to hospital admission was 3.5 days in the ICU patients and 2.1 days in the non-ICU patients $(P=0.014)$ (Table 2$)$. Thirty-three percent of the ICU group presented to the hospital within 48 hours of their onset of illness; $75 \%$ of the non-ICU group presented within 48 hours $(P=$ 0.03). All of the 15 ICU patients had abnormal chest 
TABLE 2: Clinical presentations of $2009 \mathrm{H} 1 \mathrm{~N} 1$ influenza infection.

\begin{tabular}{|c|c|c|c|c|}
\hline Clinical symptoms and signs & Total $(n=31)$ & ICU $(n=15)$ & Non-ICU $(n=16)$ & $P$ value \\
\hline Symptom days before admission & 2.8 & 3.5 & 2.1 & 0.01 \\
\hline Admission within $48 \mathrm{hr}$ of onset & $17(55 \%)$ & $5(33 \%)$ & $12(75 \%)$ & 0.03 \\
\hline Fever & $22(71 \%)$ & $12(80 \%)$ & $10(63 \%)$ & Ns \\
\hline Myalgia & $7(23 \%)$ & $4(27 \%)$ & $3(19 \%)$ & Ns \\
\hline Running nose & $19(61 \%)$ & $12(80 \%)$ & $7(44 \%)$ & Ns \\
\hline Dyspnea & $24(77 \%)$ & $14(93 \%)$ & $10(63 \%)$ & Ns \\
\hline Cough & $22(71 \%)$ & $11(73 \%)$ & $11(69 \%)$ & Ns \\
\hline Sputum production & $18(58 \%)$ & $11(73 \%)$ & $7(44 \%)$ & Ns \\
\hline Diarrhea & $4(13 \%)$ & $1(7 \%)$ & $3(19 \%)$ & Ns \\
\hline Respiration $>20$ & $15(48 \%)$ & $11(73 \%)$ & $4(25 \%)$ & 0.01 \\
\hline Heart rate $>100$ & $20(65 \%)$ & $13(87 \%)$ & $7(44 \%)$ & 0.02 \\
\hline Systolic blood pressure $<100$ & $5(16 \%)$ & $4(27 \%)$ & $1(6 \%)$ & Ns \\
\hline Oxygen saturation $<95$ & $21(68 \%)$ & $13(87 \%)$ & $10(63 \%)$ & Ns \\
\hline
\end{tabular}

Ns: nonsignificant. $P>0.05$ for comparison of ICU and non-ICU patients by $\chi^{2}$ test or Fisher's exact test.

TABLE 3: Diagnostic findings of 2009 H1N1 influenza infection.

\begin{tabular}{|c|c|c|c|c|}
\hline Laboratory & Total $(n=31)$ & $\mathrm{ICU}(n=15)$ & Non-ICU $(n=16)$ & $P$ value \\
\hline Hemoglobin $<10$ & $5(16 \%)$ & $2(13 \%)$ & $3(19 \%)$ & Ns \\
\hline White blood cell $>11,000$ & $6(19 \%)$ & $5(33 \%)$ & $1(6 \%)$ & Ns \\
\hline White blood cell $<5,000$ & $10(32 \%)$ & $2(13 \%)$ & $8(50 \%)$ & Ns \\
\hline Neutrophil > 85\% & $13(42 \%)$ & $9(60 \%)$ & $4(25 \%)$ & Ns \\
\hline Platelet $<150,000$ & $4(13 \%)$ & $3(20 \%)$ & $1(6 \%)$ & Ns \\
\hline Sodium $<135$ & $21(68 \%)$ & $10(67 \%)$ & $11(69 \%)$ & Ns \\
\hline Bicarbonate $<24$ & $20(65 \%)$ & $9(60 \%)$ & $11(69 \%)$ & Ns \\
\hline Alanine aminotransferase $>40$ & $9(29 \%)$ & $4(27 \%)$ & $5(31 \%)$ & Ns \\
\hline Abnormal chest X-ray & $20(65 \%)$ & $15(100 \%)$ & $5(31 \%)$ & $<0.001$ \\
\hline Interstitial infiltrates & $18(58 \%)$ & $15(100 \%)$ & $3(19 \%)$ & $<0.001$ \\
\hline Lobar infiltrates & $11(55 \%)$ & $7(47 \%)$ & $4(25 \%)$ & Ns \\
\hline Infiltrates within $48 \mathrm{hr}$ of admission & $12(39 \%)$ & $9(60 \%)$ & $3(19 \%)$ & 0.03 \\
\hline Positive rapid influenza test & $6(19 \%)$ & $3(20 \%)$ & $3(19 \%)$ & Ns \\
\hline Positive RT-PCR from NP swap & $28(90 \%)$ & $12(80 \%)$ & $16(100 \%)$ & Ns \\
\hline Positive blood culture & $7(23 \%)$ & $2(13 \%)$ & $5(31 \%)$ & Ns \\
\hline Positive sputum culture & $12(39 \%)$ & $5(33 \%)$ & $7(44 \%)$ & Ns \\
\hline
\end{tabular}

Ns: nonsignificant. $P>0.05$ for comparison of ICU and non-ICU patients by $\chi^{2}$ test or Fisher's exact test.

RT-PCR: reverse transcription polymerase chain reaction, NP: nasopharyngeal.

X-rays consistent with interstitial pneumonitis (Table 3); $19 \%$ of the non-ICU patients had interstitial pneumonitis on chest $\mathrm{X}$-rays $(P<0.001)$. Infiltrates within chest $\mathrm{X}$-ray within the first 48 hours of admission were seen in $60 \%$ of the ICU patients and $19 \%$ of the non-ICU group $(P=$ $0.03)$. Eighty percent of the ICU group required mechanical ventilation, and $47 \%$ required vasopressors. In logistic regression analysis, higher respiratory rate was associated with ICU admission (Table 5). Due to the small sample size and the high correlation of some of the variables with each other, we only included the four most significant variables in this analysis.

All non-ICU admissions survived the infection and were discharged; $73 \%$ of the ICU admissions survived and were discharged $(P=0.043)$. Three deaths had interstitial pneumonitis within 48 hours of admission. All of the four deaths had at least one of the three complications (ARDS, septic shock, and bacterial infections). Logistic regression analysis demonstrated that septic shock increased the likelihood of death (odds ratio $=39.2,95 \% \mathrm{CI}=1.8-857.3, P=0.02$, after adjustment for age, gender, and BMI). Ventilator use, vasopressor use, and ICU admission were not entered into the logistic regression analysis, even though they were significant factors in the univariate analysis. These clinical factors were all highly correlated with septic shock and with each other.

\section{Discussion}

Studies from several countries have reported clinical observations on hospitalized patients during the first wave in both ICU and non-ICU settings [11-15]. These studies reported 
TABLE 4: Treatment and outcomes of 2009 H1N1 influenza infection.

\begin{tabular}{|c|c|c|c|c|}
\hline Treatment and outcome & Total $(n=31)$ & $\mathrm{ICU}(n=15)$ & Non-ICU $(n=16)$ & $P$ value \\
\hline Oseltamivir treatment & $28(90 \%)$ & $14(93 \%)$ & $14(88 \%)$ & Ns \\
\hline Days from onset to oseltamivir initiation & $3.42(3.3)$ & $4.3(3.8)$ & $2.6(2.5)$ & Ns \\
\hline Oseltamivir treatment within $48 \mathrm{hr}$ of onset & $16(57 \%)$ & $6(43 \%)$ & $10(71 \%)$ & Ns \\
\hline Oseltamivir initiation before admission & $3(11 \%)$ & $2(14 \%)$ & $1(7 \%)$ & Ns \\
\hline Oseltamivir initiation on admission & $16(57 \%)$ & $8(57 \%)$ & $8(57 \%)$ & Ns \\
\hline Oseltamivir initiation within $48 \mathrm{hr}$ of admission & $4(14 \%)$ & $2(14 \%)$ & $2(14 \%)$ & Ns \\
\hline Oseltamivir initiation after $48 \mathrm{hr}$ of admission & $5(18 \%)$ & $2(14 \%)$ & $3(21 \%)$ & Ns \\
\hline Survive & $27(87 \%)$ & $11(73 \%)$ & $16(100 \%)$ & 0.04 \\
\hline Discharge to home & $20(74 \%)$ & $6(55 \%)$ & $14(88 \%)$ & 0.009 \\
\hline Discharge to long-term facility & $7(26 \%)$ & $5(45 \%)$ & $2(13 \%)$ & Ns \\
\hline ARDS & $8(26 \%)$ & $8(53 \%)$ & 0 & 0.001 \\
\hline Septic shock & $7(23 \%)$ & $7(47 \%)$ & 0 & 0.002 \\
\hline Secondary bacterial infections & $18(58 \%)$ & $8(53 \%)$ & $10(63 \%)$ & Ns \\
\hline
\end{tabular}

Ns: nonsignificant. $P>0.05$ for comparison of ICU and non-ICU patients by $t$-test, $\chi^{2}$ test, or Fisher's exact test.

ARDS: acute respiratory distress syndrome.

TABLE 5: Logistic regression analysis of risk factors for ICU admission.

\begin{tabular}{lccc}
\hline & $P$ value & Odds ratio & $\begin{array}{c}95 \% \text { confidence } \\
\text { interval }\end{array}$ \\
\hline Age & 0.54 & 0.98 & $0.90-1.05$ \\
Gender & 0.26 & 0.29 & $0.03-2.56$ \\
$\begin{array}{l}\text { Symptom days before } \\
\text { admission }\end{array}$ & 0.06 & 2.48 & $0.95-6.47$ \\
Respiratory rate & 0.006 & 1.31 & $1.08-1.58$ \\
\hline
\end{tabular}

that more than $90 \%$ of hospital admissions occurred in people younger than 65 , and most of them had at least one chronic medical condition [11-15]. Approximately 30\% of patients were obese [11-13]. Pregnancy was reported in seven to nine percent $[11-13,15]$. Bilateral infiltrates were noted on chest $\mathrm{X}$-rays on admission in $27-71 \%$ of cases [11-13]. Mortality rates were between 5\% and 17\% [11-14]. Antiviral treatment within 48 hours of the onset was identified as the only variable significantly associated with better outcomes [11]. Ninety percent of the patients admitted to our hospital were younger than 60 years, and $81 \%$ had chronic medical conditions, including chronic heart or lung diseases and obesity. The clinical data in our patients hospitalized during the second wave are similar to the information reported from the first wave in the medical literature.

The high percentage of our ICU admissions may be secondary to delayed medical care. Sixty-seven percent of patients admitted to the ICU presented after more than 48 hours of illness. Although in the logistic regression analysis $P$ value for the number of days with symptom before admission to the hospital was not below the conventional value of 0.05 , it was close $(P=0.06)$. A study of $2009 \mathrm{H} 1 \mathrm{~N} 1$ influenza infection-related ICU admissions in Canada also reported delayed medical attention in ICU patients during the first wave of the pandemic, with a median time of five days from the onset of illness to ICU admission [13]. Seventyone percent of patients in the Canadian study had bilateral infiltrates on chest X-ray on presentation. Campbell et al. reported that a delay of one day from the onset of illness to hospital admission increased the risk of death by $5.5 \%$ [14].

More patients in our ICU group received antiviral treatment after 48 hours of illness with a mean time from the illness onset to the antiviral therapy of four days in the ICU group. During the first wave of the pandemic, $79 \%$ of admissions in adult patients received antiviral treatment, but only $32 \%$ received it within 48 hours of their onset [11]. Jain et al. reported delayed antiviral treatment in patients admitted to an ICU; antiviral treatment within 48 hours of the onset was identified as the only variable significantly associated with better outcomes [11]. A Chilean study also demonstrated improved outcomes with early treatment [15]. The overall mortality rate in our study was $13 \%$. Three of four patients who died were younger than 50 years, and all had chronic medical conditions except one who was a smoker. Mortality of $2009 \mathrm{H} 1 \mathrm{~N} 1$ influenza infections during the first wave was between $5 \%$ and $17 \%$ in hospitalized patients [1114]. Jain et al. reported that all deaths were in young patients, but almost $70 \%$ of these patients had an underlying medical condition [11]. In addition, all patients who died received antiviral treatment after 48 hours of the illness onset [11].

Multiple factors potentially influence the development of successive waves during influenza pandemics. Viral transmission depends on the movement of infected source cases from communities with high levels of infection to communities with lower levels of infection. Studies in Peru have demonstrated that cases firstly occurred in large population centers and then moved to smaller communities [16]. School vacation schedules also appeared to have an important effect on the distribution of infection and the number of cases in younger age groups fell during school vacations. Consequently, schools seem to represent an initial site for the acquisition of infection with transfer into families and communities. The analysis of the spatial-temporal 
distribution of infection in Europe also suggested that the heterogeneous pattern of cases could potentially be explained by school calendars and by travel patterns [17]. The second factor in the development of successive waves involves changes in viral characteristics. Yang et al. have reported changes in the hemagglutinin gene (based on proteotyping) in H1N1 viruses during successive waves in Taiwan [18]. Consequently, immunity could fall over time if the viral antigens have sufficiently changed so that prior immunity is no longer as effective as it was once. Wessel and coworkers demonstrated that drug pressure can lead to the development of multiple waves, and, of course, the development of drug resistance makes the currently available limited antiviral therapy less effective [19]. Dang and Bauch have suggested that the timing of vaccination influences outcomes during successive waves [20]. Populations vaccinated in the late summer or the early fall may have waning immunity in the winter months. During both seasonal influenza and this pandemic more frequent ICU admissions occurred during the winter months, this resulted in poorer outcomes and more deaths. These population studies suggest that community health organizations need to provide real-time information about viral distribution, disease activity, and mortality during influenza pandemics. In particular, the timing of vaccinations and possibly revaccination in certain populations seem important. One important lesson that was available early in the $2009 \mathrm{H} 1 \mathrm{~N} 1$ pandemic is that the population at risk was in general younger than the usual population at risk in seasonal influenza, and that early treatment of sicker cases was essential to reduce morbidity and mortality.

Information collected during the first wave should have alerted clinicians to the fact that hospitalization during this pandemic largely involved young adults with chronic medical conditions or obesity and that the mortality rate for younger adults was higher than that observed in seasonal influenza infections. Antiviral treatment within 48 hours of symptom onset can decrease morbidity and mortality from influenza infection in the general population and in high-risk patients, and vaccination strategies need to consider the possibility of waning immunity during subsequent waves. Public health officials need to communicate information collected during the first wave to both the public and the health care providers and provide more information to physicians about the potential for a second wave with higher mortality. Physicians need to organize their clinical practices to provide more rapid access for patients with respiratory symptoms if a second wave starts.

\section{Conflict of Interests}

The authors declare that they have no conflict of interests.

\section{References}

[1] Centers for Disease Control and Prevention, "Updated CDC estimates of $2009 \mathrm{H} 1 \mathrm{~N} 1$ influenza cases, hospitalizations, and deaths in the United States," April 2009-April 2010, http://www.cdc.gov/h1n1flu/estimates_2009_h1n1.htm.
[2] W. W. Thompson, D. K. Shay, E. Weintraub et al., "Influenzaassociated hospitalizations in the United States," Journal of the American Medical Association, vol. 292, no. 11, pp. 1333-1340, 2004.

[3] W. W. Thompson, D. K. Shay, E. Weintraub et al., "Mortality associated with influenza and respiratory syncytial virus in the United States," Journal of the American Medical Association, vol. 289, no. 2, pp. 179-186, 2003.

[4] M. A. Miller, C. Viboud, M. Balinska, and L. Simonsen, “The signature features of influenza pandemics-implications for policy," New England Journal of Medicine, vol. 360, no. 25, pp. 2595-2598, 2009.

[5] V. Andreasen, C. Viboud, and L. Simonsen, "Epidemiologic characterization of the 1918 influenza pandemic summer wave in Copenhagen: implications for pandemic control strategies," Journal of Infectious Diseases, vol. 197, no. 2, pp. 270-278, 2008.

[6] G. Chowel, C. Viboud, L. Simonsen, M. A. Miller, and R. AcunaSoto, "Mortality patterns associated with the 1918 influenza pandemic in Mexico: evidence for a spring herald wave and lack of preexisting immunity in older populations," Journal of Infectious Diseases, vol. 202, no. 4, pp. 567-575, 2010.

[7] S. A. Richard, N. Sugaya, L. Simonsen, M. A. Miller, and C. Viboud, "A comparative study of the 1918-1920 influenza pandemic in Japan, USA and UK: mortality impact and implications for pandemic planning," Epidemiology and Infection, vol. 137, no. 8, pp. 1062-1072, 2009.

[8] J. M. Barry, C. Viboud, and L. Simonsen, "Cross-protection between successive waves of the 1918-1919 influenza pandemic: epidemiological evidence from US Army camps and from Britain," Journal of Infectious Diseases, vol. 198, no. 10, pp. 1427-1434, 2008.

[9] C. Reed, F. J. Angulo, D. L. Swerdlow et al., "Estimates of the prevalence of pandemic (H1N1) 2009, United States, apriljuly 2009," Emerging Infectious Diseases, vol. 15, no. 12, pp. 2004-2007, 2009.

[10] M. Helferty, J. Vachon, J. Tarasuk, R. Rodin, J. Spika, and L. Pelletier, "Incidence of hospital admissions and severe outcomes during the first and second waves of pandemic (H1N1) 2009," Canadian Medical Association Journal, vol. 182, no. 18, pp. 1981-1987, 2010.

[11] S. Jain, L. Kamimoto, A. M. Bramley et al., "Hospitalized patients with $2009 \mathrm{H} 1 \mathrm{~N} 1$ influenza in the United States, AprilJune 2009," New England Journal of Medicine, vol. 361, no. 20, pp. 1935-1944, 2009.

[12] S. A. R. Webb, V. Pettilä, I. Seppelt et al., "Critical care services and 2009 H1N1 influenza in Australia and New Zealand," New England Journal of Medicine, vol. 361, no. 20, pp. 1925-1934, 2009.

[13] A. Kumar, R. Zarychanski, R. Pinto et al., "Critically ill patients with 2009 influenza A(H1N1) infection in Canada," Journal of the American Medical Association, vol. 302, no. 17, pp. 1872-1879, 2009.

[14] A. Campbell, R. Rodin, R. Kropp et al., "Risk of severe outcomes among patients admitted to hospital with pandemic (H1N1) influenza," Canadian Medical Association Journal, vol. 182, no. 4, pp. 349-355, 2010.

[15] J. P. Torres, M. O'Ryan, B. Herve et al., "Impact of the novel influenza A (H1N1) during the 2009 autumn-winter season in a large hospital setting in Santiago, Chile," Clinical Infectious Diseases, vol. 50, no. 6, pp. 860-868, 2010. 
[16] G. Chowell, C. Viboud, C. V. Munayco et al., "Spatial and temporal characteristics of the 2009 A/H1N1 influenza Pandemic in Peru," PLoS ONE, vol. 6, no. 6, Article ID e21287, 2011.

[17] S. Merler, M. Ajelli, A. Pugliese, and N. M. Ferguson, "Determinants of the spatiotemporal dynamics of the 2009 h1n 1 pandemic in europe: implications for real-time modelling," PLoS Computational Biology, vol. 7, no. 9, Article ID e1002205, 2011.

[18] J.-R. Yang, Y.-P. Huang, F.-Y. Chang et al., "New variants and age shift to high fatality groups contribute to severe successive waves in the 2009 influenza pandemic in Taiwan," PLoS ONE, vol. 6, no. 11, Article ID e28288, 2011.

[19] L. Wessel, Y. Hua, J. Wu, and S. M. Moghadas, "Public health interventions for epidemics: implications for multiple infection waves," BMC Public Health, vol. 11, no. 1, article S2, 2011.

[20] U. J. Dang and C. T. Bauch, "Can interactions between timing of vaccine-altered influenza pandemic waves and seasonality in influenza complications lead to more severe outcomes?" PLoS ONE, vol. 6, no. 8, Article ID e23580, 2011. 


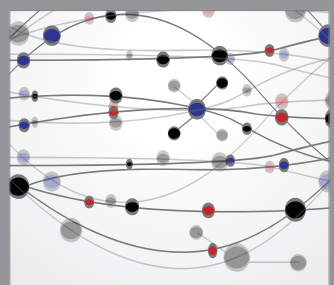

The Scientific World Journal
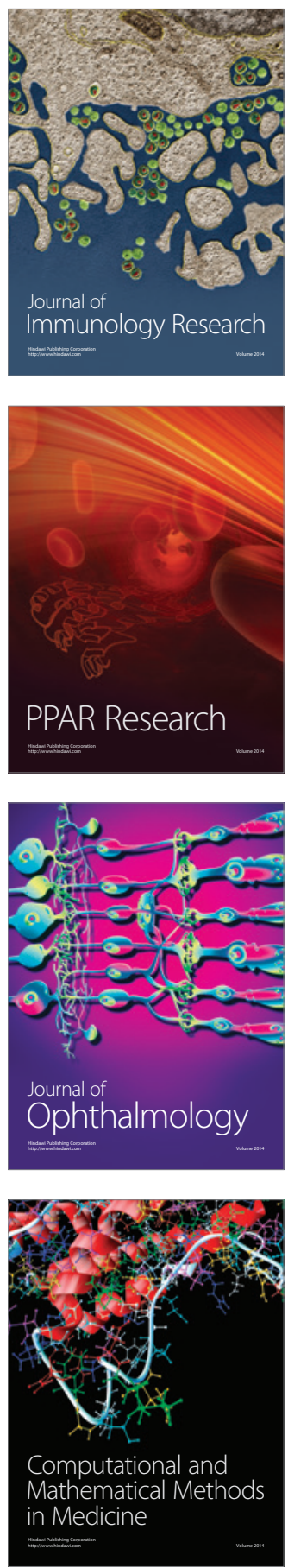

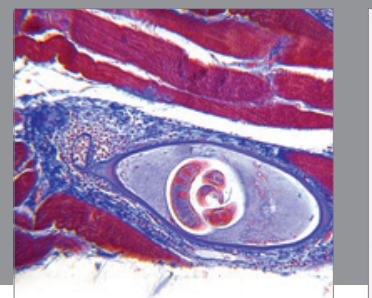

Gastroenterology

Research and Practice
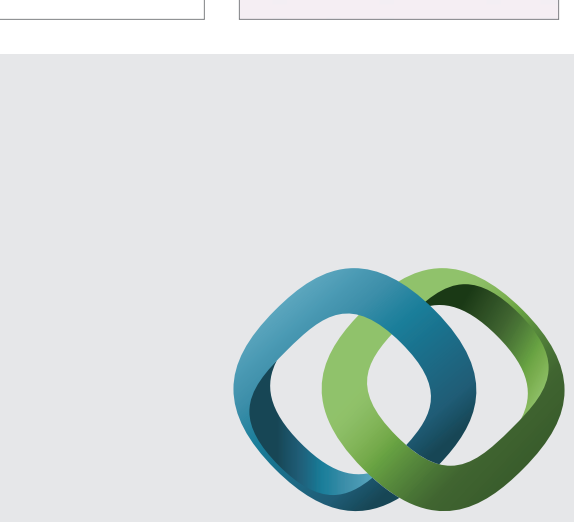

\section{Hindawi}

Submit your manuscripts at

http://www.hindawi.com
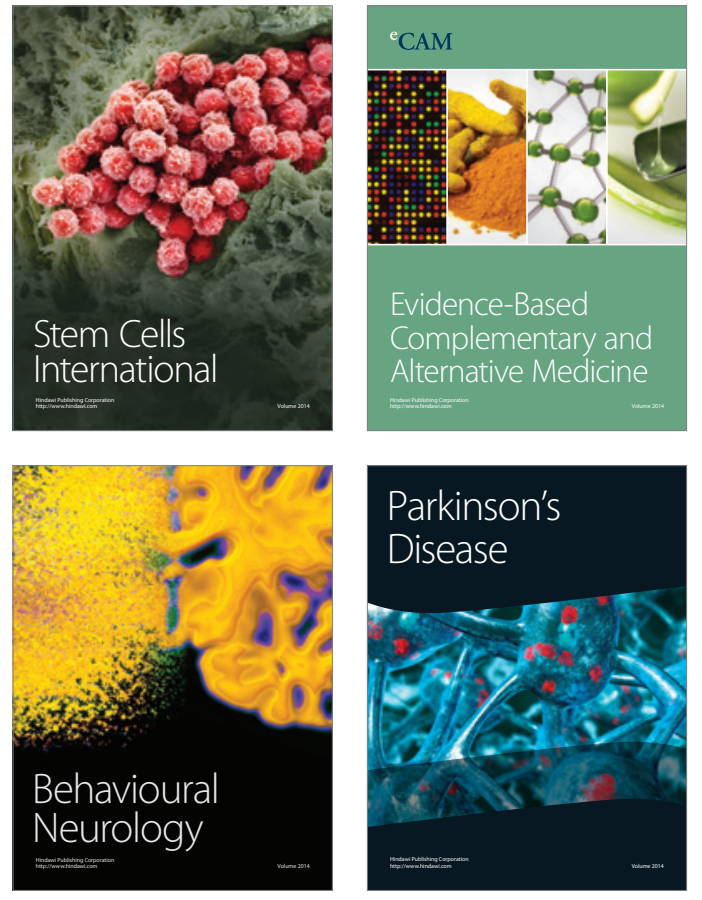
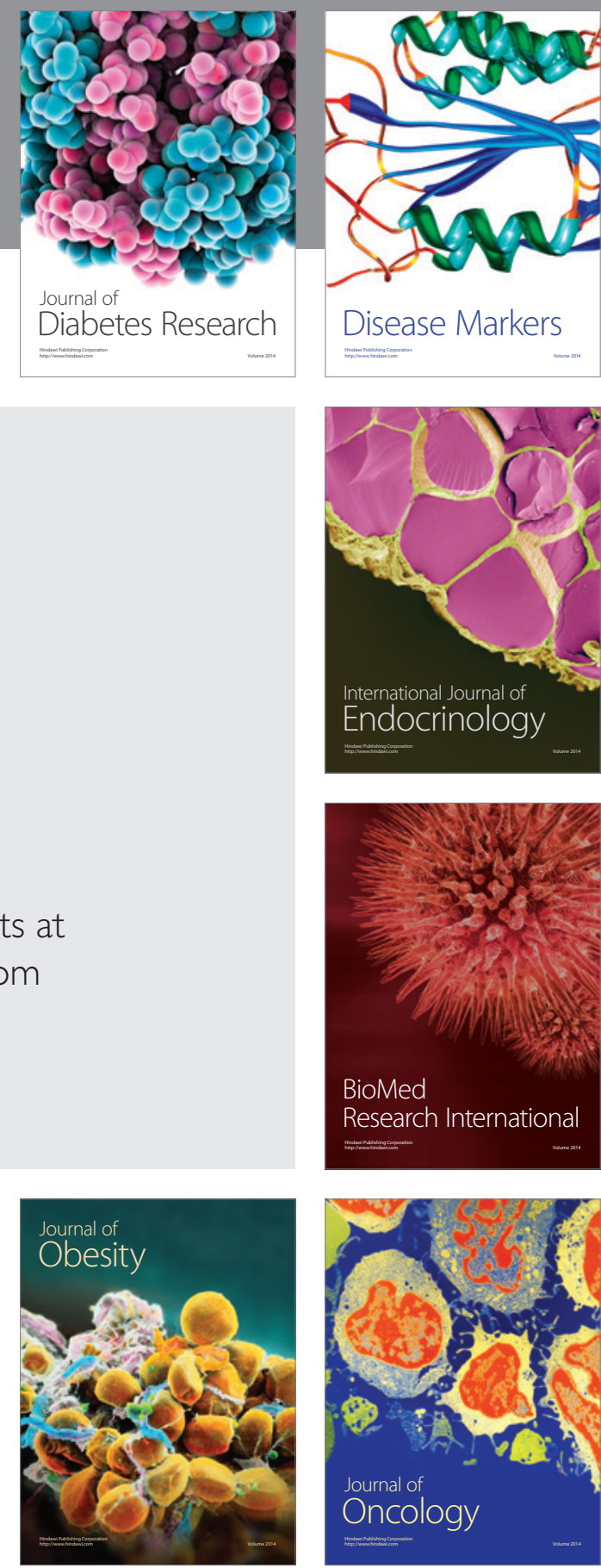

Disease Markers
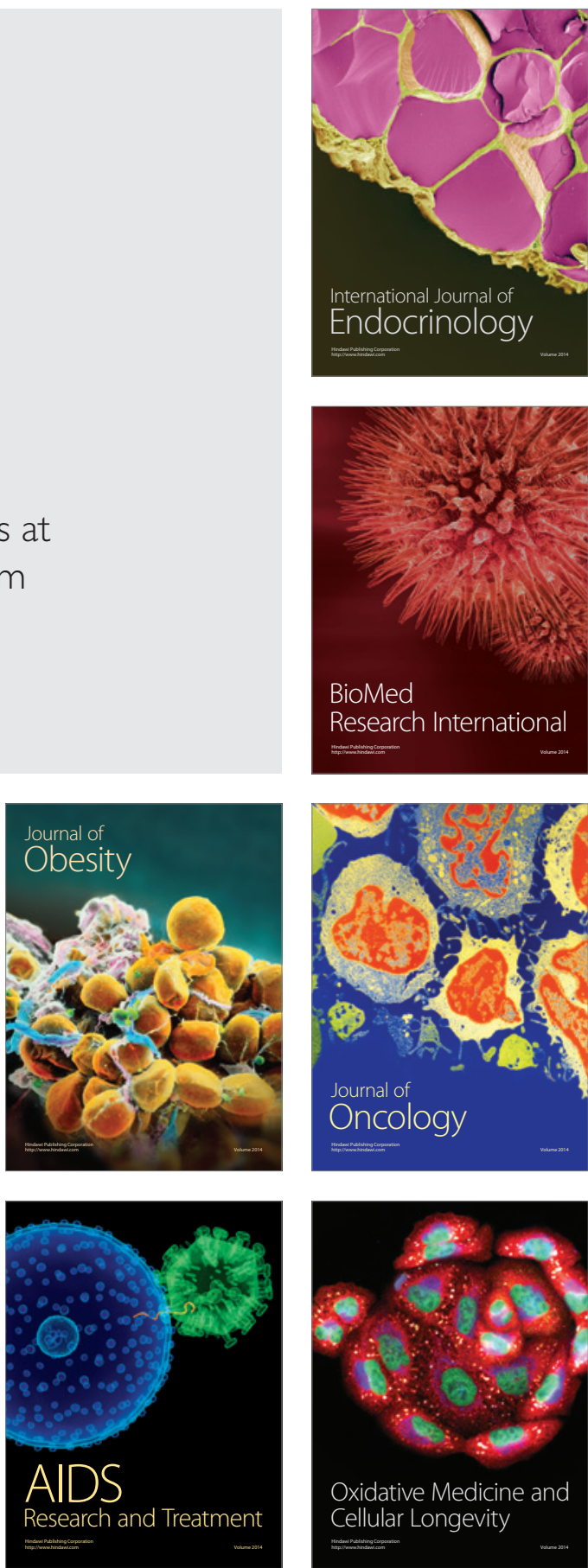